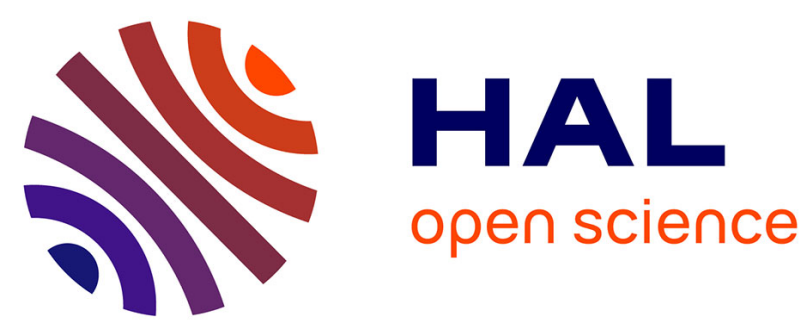

\title{
Thermal Event Recognition Applied to Protection of Tokamak Plasma-Facing Components
}

Vincent Martin, Jean-Marcel Travere, François Bremond, Victor Moncada, Gwenaël Dunand

\section{- To cite this version:}

Vincent Martin, Jean-Marcel Travere, François Bremond, Victor Moncada, Gwenaël Dunand. Thermal Event Recognition Applied to Protection of Tokamak Plasma-Facing Components. IEEE Transactions on Instrumentation and Measurement, 2010, 59 (5), pp.1182-1191. 10.1109/TIM.2009.2038032 . inria-00499599

\section{HAL Id: inria-00499599 \\ https://hal.inria.fr/inria-00499599}

Submitted on 10 Jul 2010

HAL is a multi-disciplinary open access archive for the deposit and dissemination of scientific research documents, whether they are published or not. The documents may come from teaching and research institutions in France or abroad, or from public or private research centers.
L'archive ouverte pluridisciplinaire HAL, est destinée au dépôt et à la diffusion de documents scientifiques de niveau recherche, publiés ou non, émanant des établissements d'enseignement et de recherche français ou étrangers, des laboratoires publics ou privés. 


\title{
Thermal Event Recognition Applied to Protection of Tokamak Plasma-Facing Components
}

\author{
Vincent Martin, Jean-Marcel Travere, François Brémond, Victor Moncada, and Gwenaël Dunand
}

\begin{abstract}
Magnetic confinement fusion tokamaks are complex devices where a large amount of power is required to make the fusion reactions happen. In such experimental conditions, plasmafacing components (PFCs) are subjected to high heat fluxes that can damage them. Machine protection functions must then be developed to operate current and future devices like ITER in the safest way. In current tokamaks like Tore Supra, IR thermographic diagnostics based on image analysis and feedback control are used to measure and monitor the heating of the PFCs during plasma operation. The system consists of detecting a high increase of the IR luminance signal beyond fixed qualitative levels for a set of predefined regions of interest (ROIs). The detection of overheating regions is then fully dependent on the settings of the ROIs and of the qualitative thresholds. This ROI-based approach must be improved to fit with ITER requirements and operation, where the IR scene complexity (many components monitored at the same time) will be a real challenge for the real-time PFC protection. In this paper, we propose a new vision-based approach for the automatic recognition of thermal events. This ROI-free approach, which relies on intelligent vision system concepts, is composed of two main tasks: hot spot detection and thermal event recognition. We present the results of our approach for the recognition of one critical thermal event and compare its performance with that of the previous system.
\end{abstract}

Index Terms-Fusion plasma, image and video processing, infrared (IR) imaging diagnostics, intelligent vision system, ITER, machine protection, tokamak.

\section{INTRODUCTION}

$\mathbf{M}$ AGNETIC confinement fusion is a field of research that has become more and more active since nuclear fusion has been considered as a promising approach for alternative energy production. One issue to reach a sufficient power balance in future devices like ITER is to rely on hot and long plasma produced and heated to thermonuclear temperatures by injecting large amounts of heating power (several megawatts or tens of megawatts). The goal is to inject most of the available

Manuscript received June 29, 2009; revised November 9, 2009. Current version published April 7, 2010. The Associate Editor coordinating the review process for this paper was Dr. George Xiao.

V. Martin and F. Brémond are with the Pulsar Project Team, Institut National de Recherche en Informatique et en Automatique (INRIA) Sophia Antipolis Méditerranée, 06902 Sophia Antipolis Cedex, France (e-mail: vrmartin@ sophia.inria.fr; fbremond@sophia.inria.fr).

J.-M. Travere and V. Moncada are with the Commissariat à l'Énergie Atomique/Institut de Recherche sur la Fusion Magnétique, 13108 Saint Paul-lezDurance,France (e-mail: jean-marcel.travere@cea.fr; victor.moncada@cea.fr).

G. Dunand is with the Research and Engineering Department, Sophia Conseil Society, 06560 Sophia Antipolis Cedex, France (e-mail: gwenael. dunand@cea.fr).

Color versions of one or more of the figures in this paper are available online at http://ieeexplore.iee.org.

Digital Object Identifier 10.1109/TIM.2009.2038032 power while ensuring the safety of the plasma-facing components (PFCs) by limiting the power load to just below their operational limits. In the long-pulse experiments of Tore Supra and in contrast with short-pulse experiments, the PFCs must actively be cooled down by a fast water flow, close to the plasma surface, to maintain their temperature sufficiently low to avoid melting and/or impurity pollution of the plasma (which is a unique feature of Tore Supra among other tokamaks). Any failure can lead to a water leak and time-consuming repairs. To measure and monitor the heating of the PFC during plasma operation, the most efficient way is to collect true surface temperature information using a network of IR video cameras. This is why Tore Supra, which is a carboneous device (emissivity $\sim 1$ ), is equipped with a comprehensive IR viewing system [1] made of seven endoscope bodies equipped, so far, with eight IR cameras (see Fig. 1). In the same way, the current ITER optical design [2] envisages a network of 12 IR cameras distributed in four equatorial port plugs, covering up to $70 \%$ of the vacuum chamber.

The IR camera array of Tore Supra is routinely used as input for a feedback control system based on the early detection of local hot spots to control in real time the heating power sources. More details on this unique operational control system can be found in [3].

The image analysis part of the Tore Supra feedback control consists of detecting a high increase of the IR luminance signal beyond fixed qualitative levels for a set of predefined regions of interest (ROIs) (see Fig. 2). The ROIs are defined according to the geometry of monitored objects (e.g., copper mouth and lateral protection). It has successfully been applied to the detection of some thermal events (TEs) [4] such as the electrical arcing discussed here.

Due to the increase in data volumes and scene complexity, this ROI-based approach must be improved to fit with ITER requirements and operation, as stated in [4]. Indeed, this system has several drawbacks and limitations, as listed below.

1) Working with true surface temperature values makes the strong assumption that the IR digital sensors are always well calibrated and the optical transmission factors and optical properties of observed materials (e.g., emissivity) are well known.

2) It requires an operator for: a) editing the ROI each time the PFC configuration changes or when the camera field of view has been modified after a discharge and $b$ ) tuning the qualitative thresholds (time-consuming tasks).

3) TEs that occurs outside of the ROIs cannot be detected.

4) The sensitivity to false detection is high: the presence of only one noisy or dead pixel in a ROI with an IR 


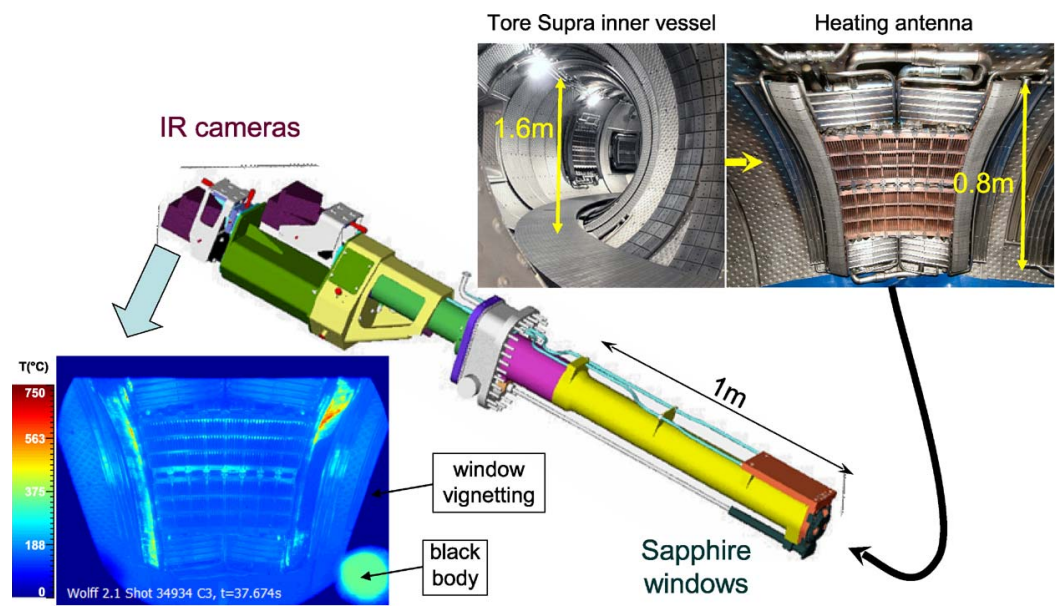

Fig. 1. Overview of one of the seven endoscopes used to monitor PFCs as heating antennas.

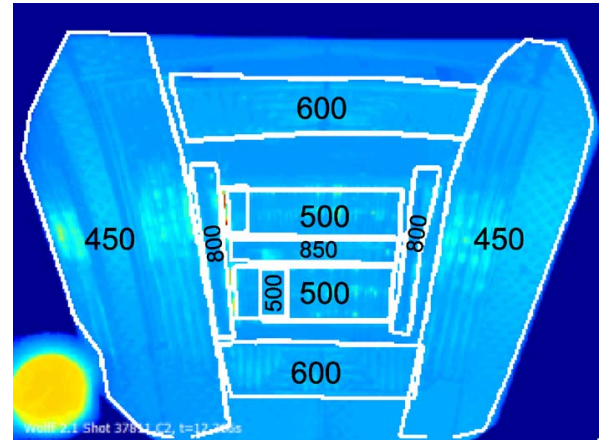

Fig. 2. ROI drawn by the user (in white) for the monitoring and the feedback control to prevent the PFCs from overheating. Qualitative thresholds on IR luminance signal are indicated for each ROI.

luminance higher than the qualitative detection threshold is enough to raise a false alarm.

5) Different TEs will not be discriminated if they occur in the same ROI.

6) Extrapolated to wide-angle view systems as planned for ITER, manual drawing of ROIs should be extremely difficult to routinely operate.

In summary, a need exists for both the improvement of the IR video processing and better understanding and interpretation of IR images as, for instance, an accurate identification of new heating zones (hot spots). In this paper, we propose a new approach for the automatic detection of TEs inspired from a video understanding framework [5] widely used in video surveillance applications.

The major advantages of such a framework compared to the current system and its previously listed issues can be justified as follows.

1) This is a qualitative image analysis approach as described in [6], relying on spatiotemporal pattern-matching techniques. The knowledge of the precise optical properties of the monitored object (e.g., emissivity of metal) is then minimized.

2) This is an ROI-free approach: the a priori knowledge of a TE is not inside the image but in the TE description model used to specialize generic image processing operators.

This paper is organized as follows. We first detail in Section II the different steps of the proposed approach for the automatic detection and recognition of TEs in IR videos. As a first step to validate this new approach, we focus on arcing event recognition in Section III. Section IV reports the recognition results on experimental data with a quantitative performance evaluation and comparison with the current system used at Tore Supra. Finally, conclusions and future perspectives are discussed in Section V.

\section{Thermal Event ReCognition}

The goal of our video understanding framework is to separate the expert knowledge of event modeling from the image processing tasks dedicated to the extraction of low-level features from visual data. To this end, we follow a bottomup approach (from pixel to semantic interpretation), which is illustrated in Fig. 3, where each of the two main vision tasks (i.e., hot spot detection and TE recognition) is specialized (e.g., parameter tuning) thanks to the formalized description of the observed TE. At the first stage (hot spot detection), we just identify localized hot spots but still not characterize them. A reasoning process is then necessary to recognize the thermal phenomena called TEs.

\section{A. A Priori Knowledge of Thermal Events}

The interpretation of observed hot spots mostly relies on a posteriori physical analysis. The goal is to explain the origin of these hot spots by the study of their temporal evolution and their localization. Some representative TEs identified during a plasma discharge are shown in Fig. 4 and correspond to the following.

1) Local RF sheath effect: Localized on the top-left corner of the Faraday screen (made of stainless steel with $B_{4} C$ coating), this hot spot is suspected to be due to the local RF electrical field carrying an enhanced power onto the antenna, as explained in [7]. The deposited power causes hot spots with the risk of melting and bursts of metallic impurities.

2) Accelerated electrons from a lower hybrid: Localized on the left-side protection (made of graphite tiles), this hot spot is suspected to be due to electrons accelerated in the near field of the lower hybrid launcher that is 


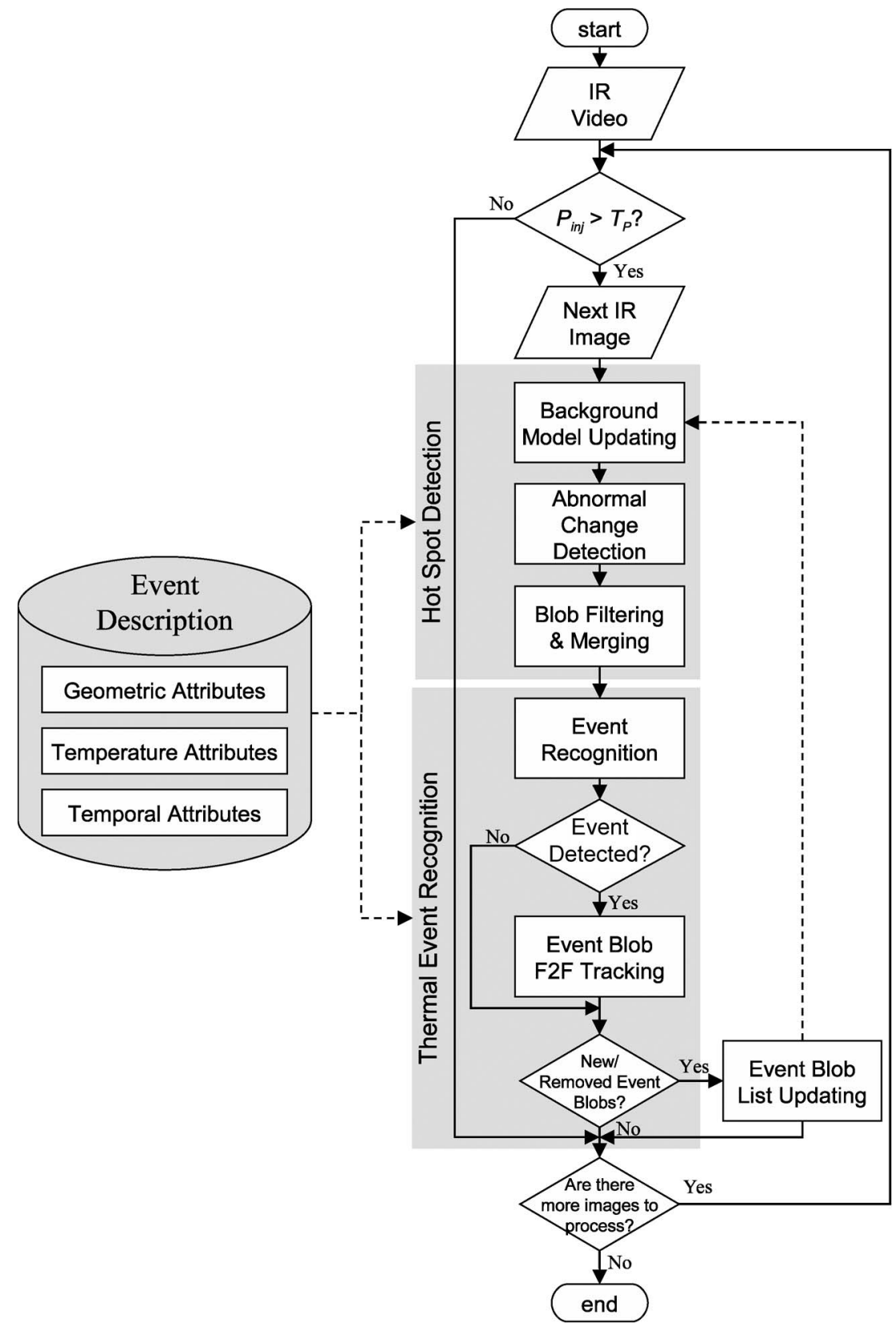

Fig. 3. Process chain of the proposed approach. The algorithm is first triggered by the value of the injected power $P_{\text {inj }}$ through a fixed threshold $T_{P}$ (here, it is fixed at $0.1 \mathrm{MW}$ ) to avoid, for instance, false detection of arcing events during plasma current ramp-up.

magnetically connected to the ion cyclotron resonance heating (ICRH) antenna, as explained in [8].

3) Fast ion losses: Localized on both the side protection and the vertical edge of the Faraday screen, it might be caused by fast ion losses created during the ICRH hydrogen minority heating scheme, as explained in [9]. In Tore Supra, these losses are an important cause of concern for the long-pulse capability at high RF power levels due to high magnetic ripples (see [10]).

4) $B_{4} C$ flakes: The hot spot has a small size and is localized on the vertical edge of the Faraday screen. It is due to the flaking of the $B_{4} C$ coating and, consequently, to the heating caused by fast ion losses, as shown in [11]. IR luminance may overpass the acceptable threshold without apparent risk of damage.
5) Electrical arcs: They are caused by a potential difference between the two lateral shields of a heating antenna during high-power discharges and can lead to the destruction of sensitive parts like the copper mouth of the antenna.

6) Specular/diffuse reflection area of the limiter on the metallic component (see [12]): This may induce false detection.

\section{B. A Priori Knowledge Formalization}

We use a formalized visual description of the TEs to detect as a support of reasoning mechanisms. This description relies on a visual concept ontology composed of generic spatiotemporal attributes such as the geometric and temporal cues introduced in [13]. The main advantage in using such a symbolic 

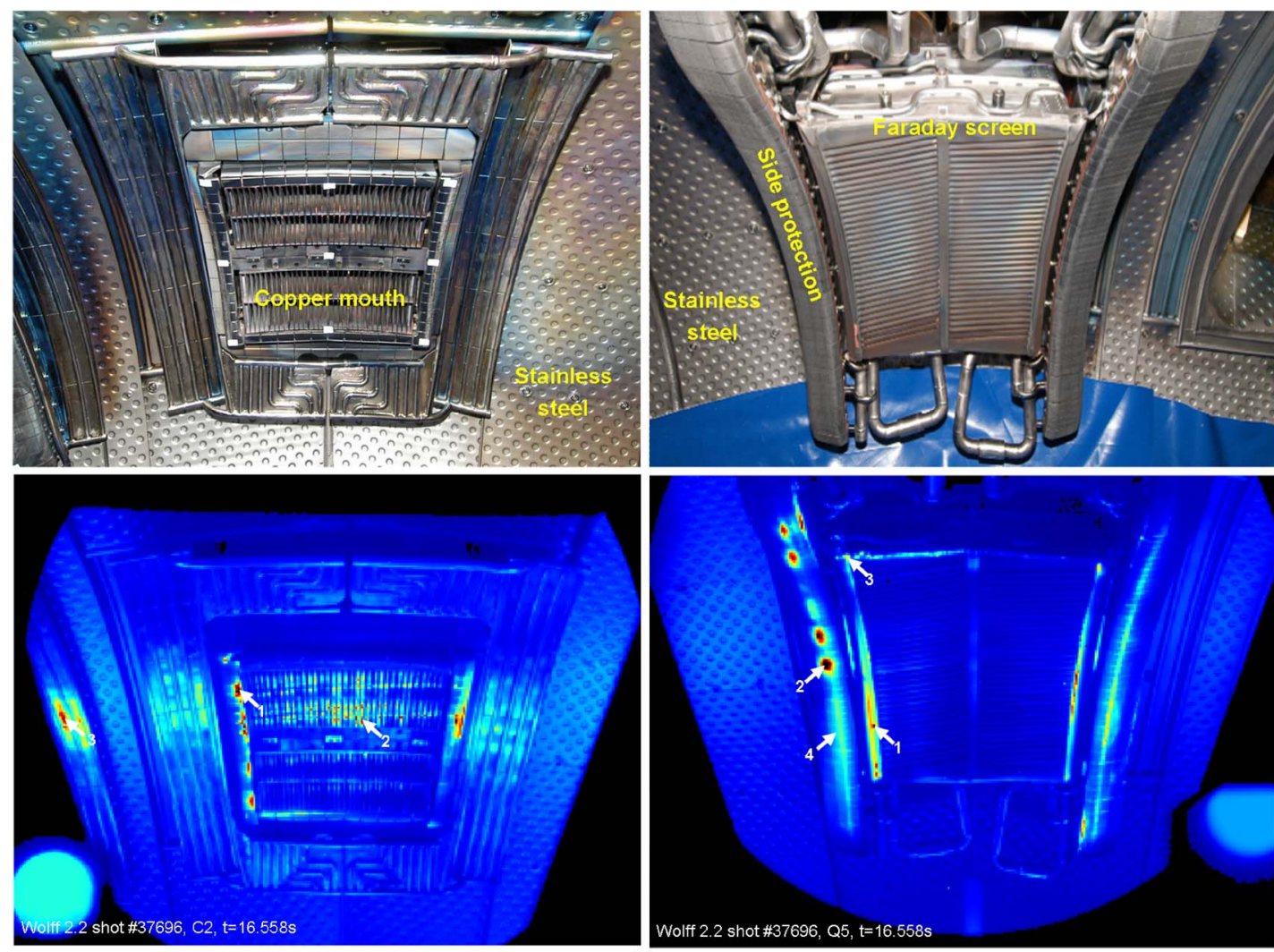

(a)


(b)

Fig. 4. Time traces of some identified hot spots (picked from the IR frames) and of the power injection (bottom plots) during a plasma discharge for two heating antennas. (a) Identified hot spots on two heating antennas Left column: Lower hybrid current drive (LHCD). Right column: ICRH antenna. (b) Time traces of the apparent temperature of the picked pixels in (a). Left: LHCD hot spots. Right: ICRH hot spots.

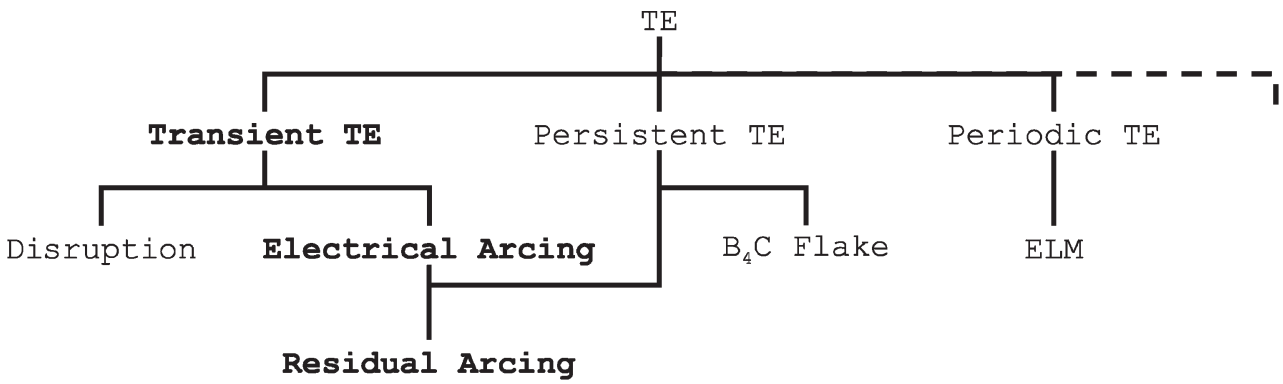

Fig. 5. Domain class hierarchy representing the different abstraction layers used to characterize the different TEs. The associated knowledge representation of TEs in boldface is described in Fig. 6.

description is its reusability in different experimental contexts. For instance, the description of a TE does not change if the image acquisition system is modified. Indeed, visual concepts are an intermediate level that helps in mapping low-level numerical values to a domain class description. We detail in Fig. 5 the TE class hierarchy that should be considered as an extendable basis. This hierarchy and the knowledge associated with each class have been defined by physicists. 


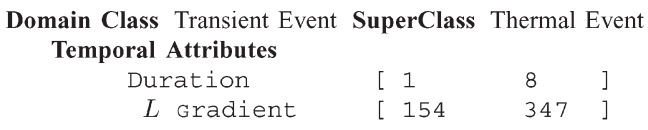

Domain Class Electrical Arcing SuperClass Transient Event Spatial Attributes

$\begin{array}{llll}\text { Length } & {[50} & 110 & ] \\ \text { Height } & {[7} & 20 & ] \\ \text { Elongation } & {[2.5} & 16 & ] \\ \text { Area } & {[} & 2200 & ]\end{array}$

Infrared Luminance Attributes

Local contrast [ $1.3 e-4 \quad 0.17$ ]

entropy [ $\left.\begin{array}{ll}3.8 & 9.02\end{array}\right]$

Domain Class Residual Arcing SuperClass Electrical Arcing and Residual TE

Domain Class Residual TE SuperClass Thermal Event Temporal Attributes

Duration [ 15 Inf ]

Fig. 6. High-level description of arc and residual arc events through the different abstraction layers defined in Fig. 5. For simplification purposes, spatial attributes have been translated from physical dimensions to pixel values, and temporal attributes have been translated from seconds to frames. Range values correspond to the observed minimum and maximum.

\section{Automatic Arcing Event Recognition}

\section{A. Arcing Event Knowledge Formalization}

For the specific case of electrical arcing, physicists describe them through their shape and their specific IR luminance dynamic. Arc shape and luminance ranges are two discriminative characteristics that can be mapped into low-level features to be extracted from visual data. The corresponding knowledge representation in terms of spatiotemporal attributes is given in Fig. 6. Range values associated with each attribute have manually been learned on a set of representative TE samples.

The visual attributes have been chosen for their low computational cost. The local contrast of a region $r$ is defined as the ratio $\left|\mu_{r}-\mu_{n r}\right| /\left(\mu_{r}+\mu_{n r}\right)$, where $\mu_{r}$ is the mean value of the pixels in region $r$, and $\mu_{n r}$ is the mean value of the pixels surrounding region $r$. The $L$ gradient is the positive variation of the IR luminance $\Delta L=L_{i}-L_{i-1}$ in the time interval $\Delta t=t_{i}-t_{i-1}$.

\section{B. Transient Hot Spot Detection}

Motion is a particularly important cue for object detection in image sequences. Indeed, a moving object is often referred as an object of interest and can then be classified as foreground. The simplest approach to detect a moving pixel in image sequences consists of subtracting the current frame from a reference frame. Since the background is rarely stationary, the key issue for any background subtraction algorithm is to efficiently model the background and update it according to the pixel variations. Many approaches have been proposed for background modeling based on unimodal distribution, mixture of Gaussians, or compressed models (see [14] for an overview). Here, the method of Butler et al. [15] is used. This algorithm has a low computational cost and is adapted to multimodal backgrounds. The basic idea of this method is to maintain some limited but important information about the history of

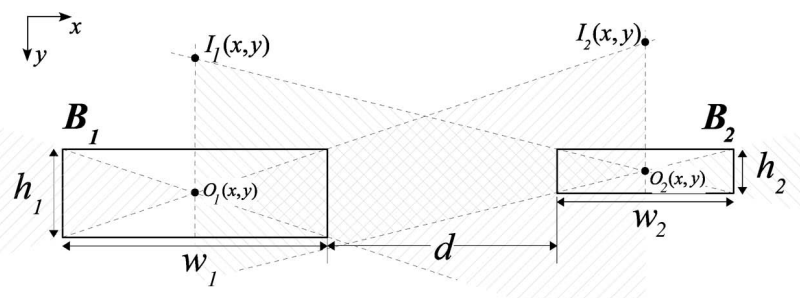

Fig. 7. Blob features $(w, h, o)$ and interblob features $(d, I)$ used by the merging criteria.

each pixel. To this end, the algorithm models each pixel in the frame by a group of clusters, where each cluster consists of an average pixel value called the centroid with an associated weight representing the cluster proportion among the others. The clusters are sorted in order of the likelihood that they model the background, and their weights and centroids are adapted to deal with background changes. An incoming pixel is then classified into a background/foreground pixel with regard to the weight of the matched cluster. The algorithm depends on three main free parameters: the number of clusters, the adaptation rate, and the foreground classification threshold. The adaptation rate is directly linked up to the thermal evolution of the TEs to detect. A small rate will be adapted for the detection of slow TEs, whereas a high rate will be adapted for the detection of transient TEs such as electrical arcing. Therefore, the setting of this parameter directly depends on the a priori knowledge given by the experts. In the same way, the foreground classification threshold can be connected to the IR luminance attributes. Basically, the parameter is set according to the local contrast attribute of the TE. In the case of arcing events, we estimated this parameter according to the average contrast value computed from a set of identified arcing events. Concerning the number of clusters, we observed only small detection differences while varying the value (between two and five), so we decided to set it to three to maintain a good tradeoff between the sensitivity and the frame rate.

\section{Arc Pattern Reconstruction}

Electrical arcs are horizontally oriented (in the video camera referential) with a strong IR signature at the copper mouth level. Nevertheless, it is very rare to observe the entire pattern in the IR images. Most of the time, only a collection of pattern subparts is visible and detected in the form of several blobs by the segmentation algorithm. This is why a specific merging process is necessary to reconstruct the whole arcing pattern.

According to the symbols used in Fig. 7, we have defined a blob-merging criterion $C$ as follows:

$$
C=c_{1} \wedge c_{2}
$$

where $\forall i, j=1,2, i \neq j$

$$
\begin{aligned}
& c_{1}=\left\{\left|y_{O_{i}}-y_{O_{j}}\right|<\left|y_{I_{i}}-y_{O_{j}}\right|\right\} \\
& c_{2}=\left\{w_{1}+w_{2}<\frac{1}{2} \times\left|x_{O_{i}}-x_{O_{j}}\right|\right\} .
\end{aligned}
$$


This criterion favors the merge of blobs having the same orientation (here, horizontal for the arcs) and capable of seeing each other in this orientation. Two blobs $B_{1}$ and $B_{2}$ are defined as visible for each other if the center of $B_{1}$ (respectively, $B_{2}$ ) is inside the hatched zone formed by $B_{2}$ (respectively, $B_{1}$ ), as seen in Fig. 7. The visibility is proportional to the inverse of the distance, to the sizes, and to the horizontal elongations of the two blobs (parameters $d, w$, and $h$ ). This criterion prevents distant small blobs from merging. Then, a new bounding box including the two merged blobs is computed, and the merging process is repeated until no more merges are possible.

\section{Arcing Event Recognition}

Once each blob has been filtered/merged, the next task is to look for evidence of given described events from the detected transient hot spots. To this end, we also rely on the a priori knowledge of Fig. 6. In a depth-first search procedure, each hypothesis that leads to the recognition of a TE is tested. The goal is to traverse the domain class hierarchy from the most abstract class (top of the tree) to the targeted TE. If the test fails at one specific layer, the recognition procedure stops at the previous layer. For the specific case of an arcing event (third layer of our TE class hierarchy), we use three criteria for event recognition:

1) pattern duration (transient event layer);

2) pattern shape (electrical arcing layer);

3) pattern size (electrical arcing layer).

The recognition algorithm verifies that all attribute values extracted from the merged blobs are within the corresponding learned values. We observe that geometric attributes prevail, most of the time, upon the other attributes (i.e., IR luminance and temporal attributes) for the arcing event recognition. In the same way, the discrimination between arcs and residual arcs (i.e., arcs lasting more than $300 \mathrm{~ms}$ ) is based on arc pattern duration. To this end, arc patterns must be tracked, as explained in the next section.

\section{E. Arcing Event Tracking}

If a new electrical arcing pattern is recognized, an identifier is associated with the corresponding bounding box, and the 2-D coordinates of the pattern are stored in a buffer. Then, at each frame, newly detected arc patterns are matched with the ones present in the buffer. The matching criterion is based on the minimal vertical distance between the two centers of the pattern-matching candidates (here, it was set at 10 pixels). Indeed, we have observed that arc patterns often occur at specific levels related to the geometry of the antenna mouth. Each of these levels has a height of approximately 20 pixels, so a distance criterion of 10 pixels prevents arc patterns located at two different levels from merging. Once an arc pattern has been recognized, we trigger the background model updating of the detected pixels in the corresponding bounding box so that a possibly residual arc pattern is not integrated into the background model (cases of missed detection in [16]). The trigger action consists of setting two parameters of the background model update algorithm, namely, the learning rate and the matching threshold parameters, to lower values. The parameters are automatically set to initial values when the arc pattern disappears.

\section{RECOGNITION RESUlTS}

\section{A. Performance Evaluation of the Proposed Approach}

To assess the performance of our approach, we have compared the results of the arcing event recognition with validation data obtained from manual annotations of a pulse data set. The pulse data set used for this evaluation is composed of 50 IR films of two heating antenna views, corresponding to a total plasma time of $1496 \mathrm{~s}$ with a discharge duration between 5 and $62 \mathrm{~s}$. The pulses have been chosen over the overall database to represent all plasma scenarios for which arcs are used to be observed. Only nine pulses do not contain any arcing events. A total of 197 arcing events have been annotated by three human subjects trained to recognize arcing events in IR videos. Fig. 8 presents qualitative results for three representative cases.

Counting results are reported in Table I. A true positive corresponds to a detected arc that is present in the annotation base. A false negative corresponds to an arc that is not detected but present in the annotation base. A false positive corresponds to a detected arc that is not present in the annotation base.

We have identified two sources of explanation for the falsenegative results.

1) The annotation is sometimes ambiguous when arcs are very close in time or when the IR signal is not sufficiently clear enough to visually identify an arcing event.

2) Most of the false-negative results correspond to borderline cases, i.e., when the arcs are very thin and/or weak. In this case, the pattern reconstruction algorithm often fails in merging the blobs (see Fig. 9). One improvement should be the triggering of the foreground classification parameter by the injected power signal, since weak arcs occur during low-power discharges only.

The false-positive cases during plasma current ramp-up mentioned in [16] have been resolved thanks to the trigger of detection and recognition algorithms by the injected power signal.

Fig. 10 presents an example of a residual arc recognition.

\section{B. Performance Comparison With the Arc Detection Algorithm Used at Tore Supra}

The algorithm used at Tore Supra for arcing event detection is based on a weighted running average over two successive frames (at times denoted as $t$ and $t-1$ ) on the maximum IR luminance (denoted as $\Delta L$ ) extracted from the union of two ROIs defined for electrical arcing (see Fig. 2). The computation of the IR luminance threshold $\tau$ is triggered by the injected power denoted as $P_{\text {inj }}$. The output is a binary signal denoted as $S_{\mathrm{ARC}}$ such as

$$
S_{\mathrm{ARC}}(t)=(\Delta L(t)>\tau(t)) \wedge\left(I_{p}(t)>0.1\right)
$$



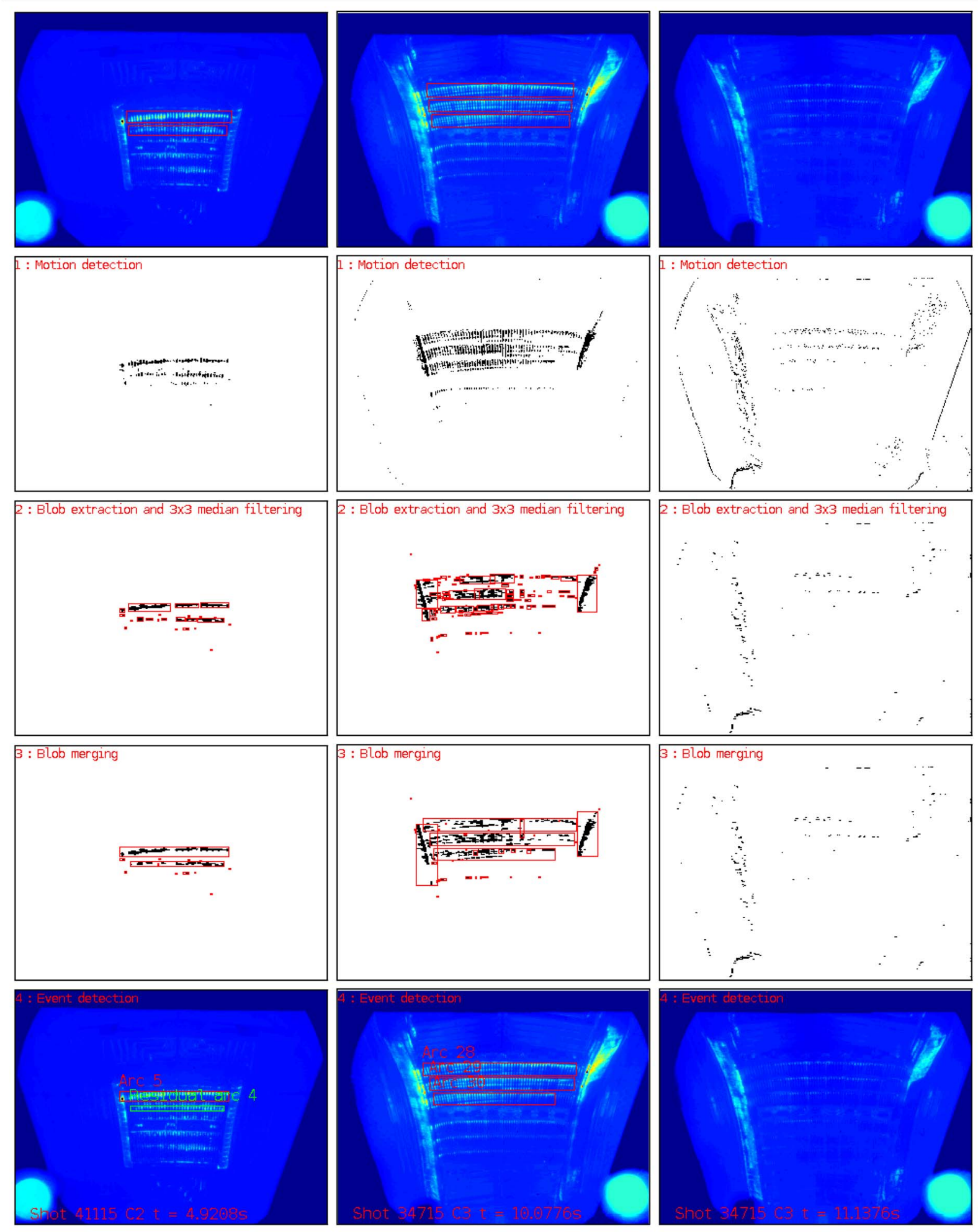

Fig. 8. Top: Ground truth with the corresponding bounding box superimposed on the original images. Row 1: Process outputs after transient change detection, detected pixels are in black. Row 2: Median filtering and blob extraction. Row 3: Pattern reconstruction. Bottom: Arcing event detection with corresponding bounding box superimposed on the original images.

where $I_{p}$ is the plasma current, and

$$
\begin{aligned}
\Delta L(t) & =L(t)-\frac{3 \times L(t-1)+L(t)}{4} \\
\Delta P_{\mathrm{inj}}(t) & =P_{\mathrm{inj}}(t)-\frac{3 \times P_{\mathrm{inj}}(t-1)+P_{\mathrm{inj}}(t)}{4}
\end{aligned}
$$

$$
\begin{aligned}
\tau(t) & =\alpha\left(1+\alpha \Delta P_{\text {inj }}(t)\right) \\
\text { with } \quad \alpha & =30 .
\end{aligned}
$$

Table II summarizes the detection results of this algorithm together with the results of the proposed approach. Since $S_{\mathrm{ARC}}$ 
TABLE I

Arcing Event Recognition Results Obtained With the Proposed Approach With Corresponding Ground-Truth (GT), True-Positive (TP), False-Negative (FN), And False-Positive (FP) Counts

\begin{tabular}{lccccc}
\hline Antenna & no. of & Annotated & \multicolumn{3}{c}{ no. of detected arcing events with our approach } \\
\cline { 3 - 6 } & pulses & arcing events & TP & FN & FP \\
\hline C2 & 28 & 140 & 133 & 7 & 0 \\
C3 & 22 & 57 & 49 & 8 & 2 \\
C2 + C3 & 50 & 197 & 182 & 15 & 2 \\
\hline
\end{tabular}

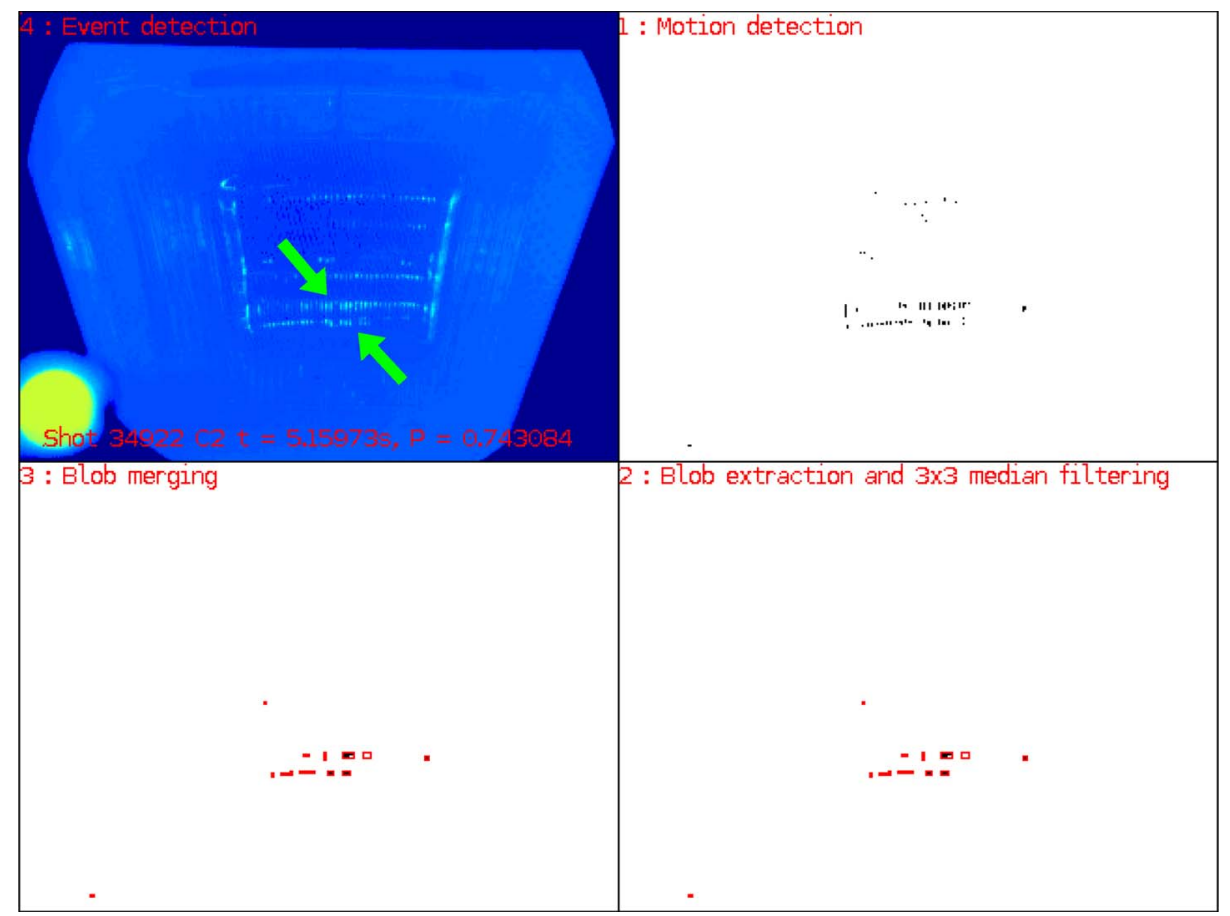

Fig. 9. One example of typical missed detection. This is a borderline case when the arc is very weak (low power injection). Despite the quite good detection, the algorithm fails to reconstruct the arc pattern due to too small and unfavorably oriented blobs.


Fig. 10. Residual arc event (i.e., arc lasting more than $300 \mathrm{~ms}$ ) detected by our approach (right) after the successful tracking of the same arc pattern \#0 (left) during $740 \mathrm{~ms}$.

is not available for all pulses of the data set used in Table I, the test data set has been reduced to 18 IR films, where 90 arcing events have been annotated, still representing a good sampling of the different scenarios.

Our proposed approach outperforms the current arc detection algorithm, particularly in terms of false detection. This large difference makes evidence of the brittleness of the thresholding technique of the current system. The practical consequence of using the proposed approach for real-time control of the launched power would be a better optimization of the power injection.

\section{Computational Performance}

In the perspective of a real-time implementation, we have assessed the computation speed of the proposed approach. The IR videos are acquired at 50 frames/s. The image size is $320 \times$ 240 pixels. The frame rate of the $\mathrm{C}++$ software implementation 
TABLE II

Comparison Between the Arcing Event Detection Results Obtained With the CurRent System Used at Tore Supra AND With the Proposed ApProach In BoldFace

\begin{tabular}{lccccc}
\hline Antenna & no. of & Annotated & \multicolumn{3}{c}{ no. of detected arcing events } \\
\cline { 3 - 6 } & pulses & arcing events & TP & FN & FP \\
\hline C2 & 11 & 73 & $68 / \mathbf{7 0}$ & $5 / \mathbf{3}$ & $51 / \mathbf{0}$ \\
$\mathrm{C} 3$ & 7 & 17 & $11 / \mathbf{1 3}$ & $6 / \mathbf{4}$ & $7 / \mathbf{0}$ \\
$\mathrm{C} 2+\mathrm{C} 3$ & 18 & 90 & $79 / \mathbf{8 3}$ & $11 / \mathbf{7}$ & $58 / \mathbf{0}$ \\
\hline
\end{tabular}

TABLE III

Computation Time For Each Step of The Proposed Approach

\begin{tabular}{l|c|c}
\hline process & processing time (in ms) & \% of the total frame rate \\
\hline detection & 41.0 & 80 \\
recognition & 3.3 & 7 \\
tracking & 6.7 & 13 \\
overall & 51.0 & 100 \\
\hline
\end{tabular}

on a $2 \times 2.33 \mathrm{GHz}$ personal computer is about 20 frames/s. As seen in Table III, the most important part of the computational time is taken by the detection algorithm. This result is obviously coherent since at this stage, the amount of information to proceed is equal to the number of pixels. Recognition and tracking algorithms have much less memory and CPU needs since they just manipulate a list of bounding boxes coded on only four values (upper left and bottom-right positions of the box). With a field-programmable gate array (FPGA) implementation of the detection algorithm, the processing should be able to deliver results every $20 \mathrm{~ms}$, i.e., at the camera frame rate.

\section{CONCLUSion And Future Work}

In this paper, we have proposed a new vision-based approach for the automatic recognition of TEs in IR images of PFCs. The proposed approach improves the current PFC monitoring system used at Tore Supra and addresses arcing event recognition. As a result, the false-detection rate is drastically reduced. As a consequence of real-time control, the power injection should be better optimized. Moreover, this ROI-free approach is independent of any displacement inside the camera field of view with a better localization of the observed TEs. This approach is going to be extended to the recognition of other TEs (e.g., $B_{4} C$ flakes, accelerated electrons, and fast particle losses) to prove its genericity. A 3-D scene model will be added to take into account, at each step of the TE recognition process, the geometrical description of the monitored objects and their optical properties. The ultimate goal of this project is to improve the reliability of the current real-time control acting on the heating sources. To this end, the hot spot detection algorithm will be implemented on an FPGA to reach realtime constraints, and the system will be tested during Tore Supra plasma operation. This will be a good starting point toward a real-time automatic feedback control system based on intelligent signal and image processing applied to the foreseen IR wide-angle viewing system of ITER, which will be involved in the crucial PFC protection function.

\section{ACKNOWLEDGMENT}

The authors would like to thank the reviewers for their substantial comments. This work, supported by the European Communities under the contract of Association between EURATOM and CEA, was carried out within the framework of the European Fusion Development Agreement. The views and opinions expressed herein do not necessarily reflect those of the European Commission.

\section{REFERENCES}

[1] D. Guilhem, J. L. Bondil, B. Bertrand, C. Desgranges, M. Lipa, P. Messina, M. Missirlian, C. Portafaix, R. Reichle, H. Roche, and A. Saille, "Tore-Supra infrared thermography system, a real steady-state diagnostic," Fusion Eng. Des., vol. 74, no. 1-4, pp. 879-883, Nov. 2005, Proceedings of the 23rd Symposium of Fusion Technology.

[2] S. Salasca, B. Esposito, Y. Corre, M. Davi, C. Dechelle, F. Pasdeloup, R. Reichle, J.-M. Travère, G. Brolatti, D. Marocco, F. Moro, L. Petrizzi, T. Pinna, M. Riva, R. Villari, E. De La Cal, C. Hidalgo, A. Manzanares, J. L. De Pablos, R. Vila, G. Hordosy, D. Nagy, S. Recsei, S. Tulipan, A. Neto, C. Silva, L. Bertalot, C. Walker, C. Ingesson, and Y. Kaschuck, "Development of equatorial visible/infrared wide angle viewing system and radial neutron camera for ITER," Fusion Eng. Des., vol. 84, no. 7-11, pp. 1689-1696, Jun. 2009.

[3] P. Moreau, O. Barana, S. Brémond, L. Colas, A. Ekedahl, F. SaintLaurent, C. Balorin, G. Caulier, C. Desgranges, D. Guilhem, M. Jouve, F. Kazarian, G. Lombard, L. Millon, R. Mitteau, P. Mollard, H. Roche, and J.-M. Travere, "RF heating optimization on Tore Supra using feedback control of infrared measurements," Fusion Eng. Des., vol. 82, no. 5-14, pp. 1030-1035, Oct. 2007.

[4] J.-M. Travere and Tore Supra team, "In-vessel components imaging systems: From the present experience towards ITER safe operation," Fusion Eng. Des., vol. 84, no. 7-11, pp. 1862-1866, Jun. 20093

[5] F. Brémond, M. Thonnat, and M. Zuniga, "Video-understanding framework for automatic behavior recognition," Behav. Res. Meth., vol. 3, no. 38, pp. 416-426, Aug. 2006.

[6] R. Reichle, E. dela Cal, Y. Corre, M. Davi, A. Manzanares, J. L. de Pablos, R. Vila, S. Salasca, and J.-M. Travere, "On the operational specifications and associated R\&D for the VIS/IR diagnostic for ITER," in Proc. Int. Conf. Adv. Nucl. Instrum. Meas. Methods Appl., Jun. 2009, pp. 1-6.

[7] L. Colas, L. Costanzo, C. Desgranges, S. Brémond, J. Bucalossi, G. Agarici, V. Basiuk, B. Beaumont, A. Bécoulet, and F. Nguyen, "Hot spot phenomena on Tore Supra ICRF antennas investigated by optical diagnostics," Nucl. Fusion, vol. 43, no. 1, pp. 1-15, Jan. 2003.

[8] M. Goniche, D. Guilhem, P. Bibet, P. Froissard, X. Litaudon, G. Rey, J. Mailloux, Y. Demers, V. Fuchs, P. Jacquet, J. H. Harris, and J. T. Hogan, "Enhanced heat flux in the scrape-off layer due to electrons accelerated in the near field of lower hybrid grills," Nucl. Fusion, vol. 38, no. 6, pp. 919937, Jun. 1998

[9] V. Basiuk, L.-G. Eriksson, V. Bergeaud, M. Chantant, G. Martin, F. Nguyen, R. Reichle, J. C. Vallet, L. Delpeche, and F. Surle, "Ripple losses during ICRF heating in Tore Supra," Nucl. Fusion, vol. 44, no. 1, pp. 181-192, Jan. 2004.

[10] A. Ekedahl, J. Bucalossi, V. Basiuk, S. Brémond, L. Colas, Y. Corre, E. Delchambre, D. Douai, R. Dumont, G. Dunand, G. Giruzzi, M. Goniche, S. Hong, F. Imbeaux, F. Kazarian, G. Lombard, L. Manenc, O. Meyer, L. Millon, R. Mitteau, P. Monier-Garbet, P. Moreau, B. Pégourié, F. G. Rimini, F. Saint-Laurent, F. Samaille, J. L. Schwob, and E. Tsitrone, "Operational limits during high power long pulses with radiofrequency heating in Tore Supra," Nucl. Fusion, vol. 49, no. 9, p. 095 010, Sep. 2009. 
[11] M. Chatelier, "Integration of high power, long pulse operation in Tore Supra in preparation for ITER," Nucl. Fusion, vol. 47, no. 10, pp. S579S589, Sep. 2007, on behalf of Equipe Tore Supra.

[12] D. Guilhem, R. Reichle, and H. Roche, "Reflections and surface temperature measurements in experimental fusion reactors Tore-Supra, jet and ITER," Quant. InfraRed Thermogr. J., vol. 3, no. 2, pp. 155-168, 2006.

[13] N. Maillot and M. Thonnat, "Ontology based complex object recognition," Image Vis. Comput., vol. 26, no. 1, pp. 102-113, Jan. 2008.

[14] R. J. Radke, S. Andra, O. Al-Kofahi, and B. Roysam, "Image change detection algorithms: A systematic survey," IEEE Trans. Image Process., vol. 14, no. 3, pp. 294-307, Mar. 2005.

[15] D. E. Butler, Jr., V. M. Bove, and S. Sridharan, "Real-time adaptive foreground/background segmentation," EURASIP J. Appl. Signal Process., vol. 2005, no. 1, pp. 2292-2304, Jan. 2005.

[16] V. Martin, J.-M. Brémond, F. Travere, V. Moncada, and G. Dunand, "Thermal event recognition applied to tokamak protection during plasma operation," in Proc. IEEE Int. Instrum. Meas. Technol. Conf., May 2009, pp. 1690-1694.

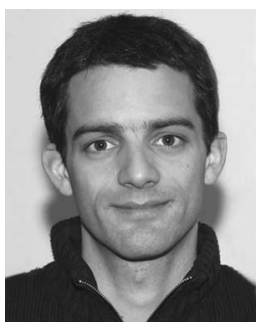

Vincent Martin received the Master's degree in astronomy and the Ph.D. degree in cognitive vision from the University of Nice Sophia Antipolis, Nice, France, in 2003 and 2007, respectively.

He joined the ORION Research Team, Institut National de Recherche en Informatique et en $\mathrm{Au}$ tomatique (INRIA), Sophia Antipolis, France. He is currently a Postdoctoral Researcher of INRIA Pulsar Project Team. He works on a collaborative project between INRIA and the Commissariat à l'Énergie Atomique, Cadarache, France, named moniTORE for Real-time Monitoring of Imaging Diagnostics Applied to Tore Plasma Operation. He has participated to various national and international collaborations in the field of video surveillance, biological and medical applications. He is the author or a coauthor of several scientific papers published in international journals and conferences in cognitive vision and related applications. His research interests include image and video processing, machine learning, and intelligent vision systems.

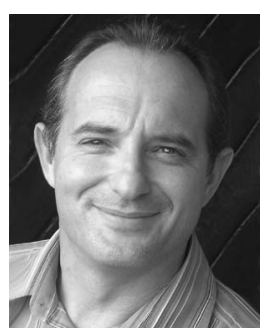

Jean-Marcel Travere received the Graduate Engineer degree from the Ecole Nationale Supérieure d'Ingénieurs de Caen et Centre de Recherche (ENSICAEN), Caen, France, in 1984 and the Ph.D. degree from Commissariat à l'Énergie Atomique (CEA), Cadarache, France, in 1987. During his Ph.D., he has worked on fully automated analysis methods applied to positron emission tomographic (PET) quantitative images and image fusion between PET images and magnetic resonance images (MRIs).

He is currently a Senior Expert in general instrumentation and image analysis with the CEA/Institut de Recherche sur la Fusion Magnétique (IFRM), Saint Paul-lez-Durance, France. He participated with the genesis of CYCERON PET Research Center, Caen, and headed the CYCERON instrumentation group for ten years. He has been involved in different European (European Concerted Action on PET) and international projects (National Institutes of Health in the U.S. and McGill Hospital in Canada) dealing with 3-D segmentation and 3-D intelligent image analysis applied to PET/MRI brain studies. He also worked on the enhancement of 2-D/3-D PET image reconstruction by the use of a priori knowledge in the tomographic reconstruction process. In parallel, he has been an expert in important French "high-tech" scientific projects: the genesis of the French national high-speed research network (RENATER) and national equipment with the new generation of 3-D PET scanners. He collaborated with international companies (SIEMENS, General Electric, PIXAR, and Silicon Graphics). In 1998, he switched to fusion research and continued to develop his expertise in instrumentation and image analysis in this new field of research. He was in charge of the Thomson Scattering system at CEA/IRFM and was with the Joint European Torus, Culham, U.K., where he worked on the LIDAR system. He headed the Imaging and Diagnostics Group at CEA/IRFM for four years to introduce intelligent image analysis concepts in the fusion community. Since 2009, he has been involved in the dissemination of this knowledge at the European level (European Fusion Development Agreement) and international level (ITER project). He has been responsible for six $\mathrm{Ph} . \mathrm{D}$. students and more than 40 postdoctoral students. He is the author or a coauthor of more than 60 scientific papers published in international journals or conference proceedings.

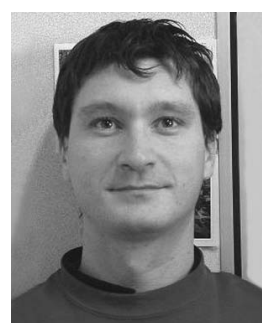

François Brémond received the Master's degree from the École Normale Supérieure de Lyon, Lyon, France, in 1992 and the Ph.D. degree in video understanding from the Institut National de Recherche en Informatique et en Automatique (INRIA), Sophia Antipolis, France, in 1997.

He was a Postdoctorate with the University of Southern California, Los Angeles, where pursued his research work on the interpretation of videos taken from unmanned airborne vehicle in the Defense Advanced Research Projects Agency (DARPA) Visual Surveillance and Activity Monitoring (VSAM) project. In 2005, he was a cofounder of Keeneo, a company in intelligent video surveillance. $\mathrm{He}$ also has participated with six European projects (PASSWORD, ADVISOR, AVITRACK, SERKET, CANTATA, and COFRIEND), one DARPA project, several national projects (SAMSIT, SIC, VideoID,...), seven industrial research contracts (RATP, FNCA, SNCF, ALSTOM, ST-MicroElectronics,...), and several international cooperations (U.S., Taiwan, U.K., and Belgium) in video understanding. He currently leads the Pulsar Project Team, INRIA Sophia Antipolis Méditerranée, Sophia Antipolis. He is the author or a coauthor of more than 75 scientific papers published in international journals or conference proceedings in video understanding. He designs and develops generic systems for dynamic scene interpretation. The targeted class of applications is the automatic interpretation of indoor and outdoor scenes observed with various sensors and in particular with static cameras. These systems detect and track mobile objects, which can be either humans or vehicles, and recognize their behaviors. He is particularly interested in filling the gap between sensor information (pixel level) and recognized activities (semantic level).

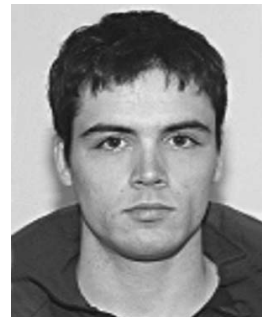

Victor Moncada received the Master's degree French-equivalent diploma on nuclear physics from Phelma, Grenoble, France, in 2007. He received an Applied Research Diploma in Sofware Engineering from the Institut National Polytechnique de Grenoble, Grenoble, France, in 2009.

Since 2008, he has been with the CEA/Institut de Recherche sur la Fusion Magnétique, Saint Paul-lezDurance, France, participating with the moniTORE project where he is in charge of software engineering activities. He is interested in designing and developing tools for imaging data analysis.



Gwenaël Dunand received the M.Sc. diploma in signal and image processing from the Institut Polytechnique de Grenoble, Grenoble, France, in 2006.

Since 2009, he has been the Research and Engineering Department, French Sophia Conseil Company, Sophia Antipolis. He is currently working for the Commissariat à l'Énergie Atomique, Cadarache, France, on the moniTORE project as an expert in software design and hardware integration to perform real-time detection of thermal events in infrared images during tokamak operation. He is interested in software engineering and real-time applications based on computer vision. 